für Holzkunde und Forstnutzung und das Institut für Forstsamenkunde und Pflanzenzüchtung; zwei seiner drei Nachfolger waren lange Jahre Assistenten in seinem Institut.

$\mathrm{Zu}$ herzlichen Glückwünschen für weitere Jahre guter Gesundheit und geistiger Frische verbinden sich mit allen ehemaligen Schülern in erster Linie seine Fakultätskollegen und seine Nachfolger auf den drei Lehrstïhlen. Der Rektor der LudwigMaximilians-Universität, Magnifizenz Professor Dr. Gerhard Weber, hat in festlichem Rahmen am 12. Januar sehr herzliche Glückwünsche an den Jubilar gerichtet und ihm eine Reihe weiterer seltener Jubiläen gewünscht. Noch 1965 werden es 60 Jahre, daß Geheimrat Fabricius als Privatdozent in den Lehrkörper unserer Universität eingetreten ist, und 25 Jahre, seit er im Status des Emeritus, aber häufig als Teilnehmer an den Fakultätssitzungen, lebt. Geheimrat Fabricius hat dem Rektor mit einer Ansprache geantwortet, die ob ihrer jugendlichen Frische und in der gewohnten Prägnanz allen Teilnehmern dauernd in Erinnerung bleiben wird. Mit dem Rektor unserer Universität wünschen und hoffen wir, daß Geheimrat FabRICIUs noch an vielen seltenen Feststunden teilnehmen wird.

J. N. KöstLer

\title{
Verleihung des Wilhelm-Leopold-Pfeil-Preises 1965
}

Der Wilhelm-Leopold-Pfeil-Preis, der von der Stiftung F. v. S. zu Hamburg zur Auszeichnung von Verdiensten um eine beispielhafte Waldwirtschaft in Europa zur Verfügung gestellt wurde, wurde für das Jahr 1965 dem Stadtforstmeister von Villingen/Schwarzwald, Forstdirektor Dr. U. Rodenwaldt, zugesprochen. Der Preis ist mit $10000,-$ DM dotiert.

Damit sollen die Verdienste RODENWALDTs, um den auf Grund wissenschaftlicher Erkenntnisse beispielhaft durchgeführten Aufbau des Stadtwaldes von Villingen und um die forstgeschichtliche Forschung, ausgezeichnet werden.

\section{BUCHBESPRECHUNGEN}

Forstwissenschaft im Dienste der Praxis. Vorträge der Forstlichen Hochschulwoche Freiburg 1961. Herausgegeben von Prof. Dr. K. MaNTEL. BLV Verlagsgesellschaft München, Basel, Wien 1962. 277 S., Format 16,5 $\times 24 \mathrm{~cm}$. Brosch. 36,- DM.

Vorträge der Forstwissenschaftlichen Hochschultagung 1962 in München. Mitteilungen aus der Staatsforstverwaltung Bayerns, 34. Heft. Bayerisches Staatsministerium für Ernährung, Landwirtschaft und Forsten - Ministerialforstabteilung - München 22, Ludwigstraße 2, 1964. 350 S., Format 15,5 × $23 \mathrm{~cm}$. Brosch. 14,- DM.

Die forstwissenschaftlichen Lehrstätten der Bundesrepublik veranstalten in jährlichem Wechsel Hochschulwochen, die den praktisch tätigen Forstleuten Inhalt und Ergebnisse der Forschungsarbeit vermitteln sollen. Von den Tagungen 1961 in Freiburg und 1962 in München sind Sammelbände mit den Texten der von Lehrkörper und Assistenten gehaltenen Vorträge erschienen. Die Vielzahl dieser Vorträge läßt eine Besprechung im einzelnen nicht zu. Es genüge der Hinweis, daß beide Bände eine Fülle wissenschaftlicher Erkenntnisse enthalten, die in vielen Fällen unmittelbar in der praktischen Arbeit anwendbar sind.

A. BERNHART 